\title{
LAS STATIONES CON AQUAE... EN LA TABULA DE PEUTINGER
}

\section{The stationes with Aquae... in the Peutinger Map}

\author{
María J. Peréx Agorreta ${ }^{1}$ \\ Jesús Rodríguez Morales ${ }^{2}$
}

Recibido el 2 de abril de 2012. Aceptado el 2 de agosto de 2012

Resumen. A partir del conocimiento de las propiedades de las aguas mineromedicinales $y / 0$ termales, y de su uso, van surgiendo, a lo largo del imperio romano, asentamientos que, en algunos casos, llegan a constituir ciudades, cuyo nombre viene definido por su origen: son las denominadas Aquae. A través tanto de las fuentes literarias, como de la epigrafía, se conocen más de un centenar de agrupaciones urbanas con este nombre que, en el caso del mapa de Peutinger, aparecen normalmente acompañadas de un símbolo característico. Palabras clave: mapa, establecimiento termal, agua medicinal, ciudad, símbolo.

Abstract. Through the knowledge of the thermal-mineral waters and their healing properties, many springs gave rise to the foundation of cities in the Roman world that were called Aquae. Literary and epigraphic sources give us more than one hundred urban settlements with this denomination that, in the Peutinger Map, has a distinct iconographic symbol.

Key words: map, thermal establishment, curative waters, city, symbol.

\section{LAS CIUDADES DE AQUAE}

Frente a los baños, cuya finalidad higiénica y lúdica es bien conocida, nos encontramos en el mundo romano con otro tipo de termas, las medicinales, con características estructurales y objetivos distintos. Aunque los romanos no emplearon un término que diferenciara unas de otras, ni aquellas en las que había que calentar el agua de las que aprovechaban el agua caliente natural, las diferencias son muy destacables. Así, mientras las termas seguian unas pautas constructivas bien conocidas, y su situación dentro de la ciudad o de la villa se planificaba en función del resto de los edificios, la situación del establecimiento termal cuyas aguas tienen propiedades curativas,al que podemos denominar balneario (o con un barbarismo muy difundido: spa), dependía para su localización de las características geológicas que determinaban la temperatura y las propiedades del agua. Sus edificaciones no seguian, por tanto, las pautas aplicables a las termas: no había que calentar el agua, sino, por el contrario, en muchas ocasiones había que enfriarla; no se desplazaba a grandes distancias para que no perdiera sus propiedades; el tamaño de las piscinas dependía de sus aplicaciones y tratamiento y, sobre todo, el balneario estaba donde se encontraba el manantial.

A partir del aprovechamiento humano de dichas aguas, surgen a lo largo del imperio romano agrupaciones urbanas denominadas Aquae, de las que se conocen casi un centenar de ejemplos. Ya Plinio el Viejo, en un párrafo muy conocido (N. H., 31, 4: 695) lo pone de manifiesto:

"(Aquae)... urbesque condunt, sicut Puteolos in Campania, Statiellas in Liguria, Sextias in Narbonensi provincia. nusquam tamen largius quam in Baiano sinu nec pluribus auxiliandi generibus ${ }^{3} . "$

(1) Departamento de Historia Antigua, UNED.mperex@geo.uned.es

(2) I.E.S. Juan Gris, Móstoles.

(3) "(Las aguas) dan origen a ciudades, como Putéolos, en Campania, Sttatiellae, en Liguria, Sextiae en la provincia Narbonense. En ninguna parte son tan abundantes como en la bahía de Bayas". 
La toponimia moderna permite identificar dichas agrupaciones urbanas fundamentalmente a través de tres denominaciones específicas: las que proceden directamente del latín (Aquae) Calidae y Balneum, y, en la península Ibérica, y en el sur y oriente del Mediterráneo, las que perviven a través del término árabe Al-Hamma.

\section{LA TABULA DE PEUTINGER}

\subsection{Descripción}

La Tabula Peutingeriana es una copia medieval -de los siglos XII o XIII- de un mapamundi, o de un itinerarium pictum romano (Levi 1967), redactado, posiblemente, en la segunda mitad del siglo IV (Miller 1962: 2). Su nombre procede de Konrad Peutinger ${ }^{4}$. Hoy se conserva en la Biblioteca Nacional de Austria de Viena. Es un rollo de pergamino de 0,34 metros de alto y 6,75 metros de largo, que fue dividido en 12 o 13 hojas o segmentos para su mejor preservación.

La primera hoja conservada ${ }^{5}$ muestra, a la izquierda un fragmento del sudeste de Britania y la zona oriental de los Pirineos y en el resto Galia y el norte de África, con caminos, ciudades y las principales islas del Mediterráneo occidental: Córcega y Cerdeña. En el segmento II continúan
Galia y África y aparece Germania en la zona superior. En el segmento III se presentan los Alpes y la Galia transalpina, con África al sur. En el IV se muestra Italia central. Se da especial importancia a Roma, con todas las calzadas que conducen a ella, formando una red radial de doce caminos, rotulados con sus nombres: Via Appia, Latrina, Labicana, etc. Una figura sentada en un trono representa alegóricamente la ciudad; su forma de presentación en la Tabula es similar a otras dos metrópolis: Constantinopla y Antioquía. El segmento $\mathrm{V}$ muestra el centro- sur de Italia, con la Bahía de Nápoles en el centro. Al norte Istria y Mesia y al sur África. En el VI segmento aparece la bota de Italia, Calabria y Sicilia y Grecia. En el VII segmento sigue Grecia y aparecen Creta y la Cirenaica al sur. En el VIII tenemos Constantinopla a la izquierda, la Península de Anatolia en el centro y el delta del Nilo abajo. En el IX, la costa de Palestina, Antioquía y Chipre. En el X Mesopotamia y en el XI y último el Mar Caspio, la península de la India y, en la esquina inferior derecha, la isla de Taprobana, nombre antiguo de Ceilán.

La mayor parte de los investigadores consideran el mapa el resultado de una compilación tardía -quizás de finales del siglo III o principios del IV d.C.- en la que conviven elementos antiguos, como la referencia a las ciudades de Pompeya, Herculano y Oplontis, sepultadas por el Vesubio en el 79 d.C.

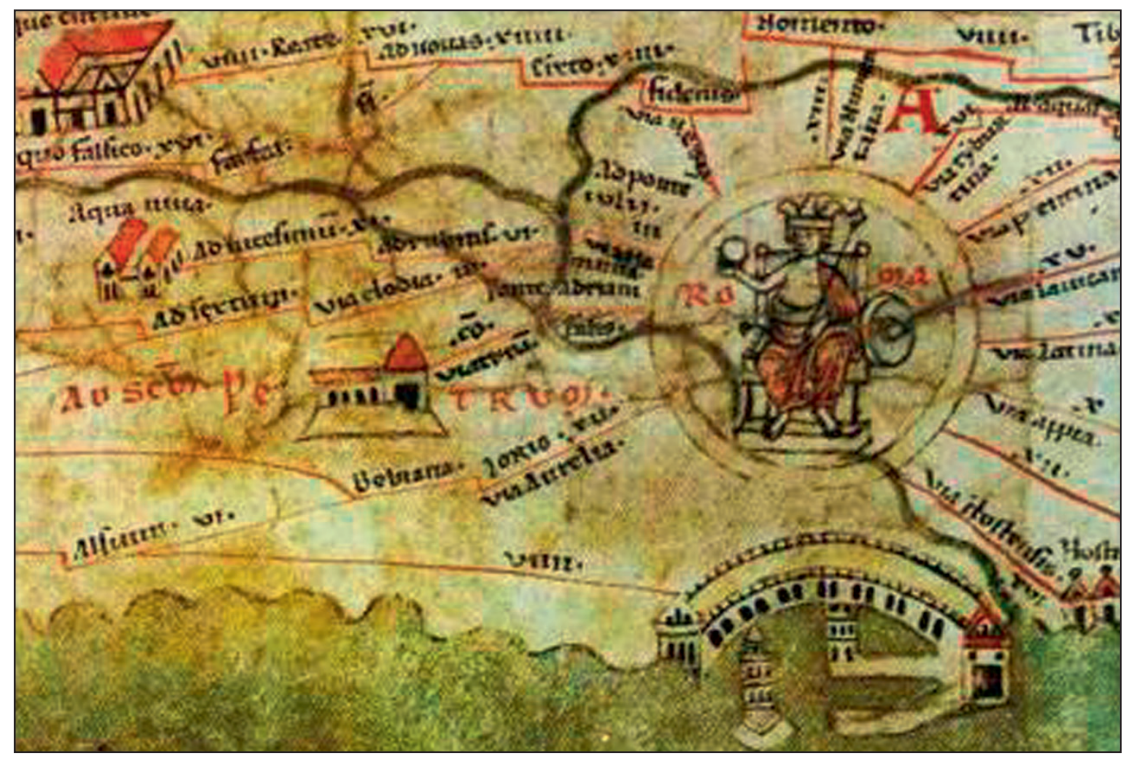

Figura 1. Tabula de Peutinger. Segmento IV: Roma.

(4) Humanista alemán, miembro del Senado de Augsburgo, a quien le fue legada por el vienés Konrad Celtes, bibliotecario del Emperador, que la encontró "en un lugar de la biblioteca", en 1508.

(5) Faltan una o dos hojas al principio, que ilustraban Britania, Hispania y Mauritania Tingitana. No obstante, tenemos documentadas enHispania las siguientes Aquae que posiblemente aparecian en la Tabula: Aquae Querquennae (Baños de Bande, Ourense), Aquae Oreginae (Rio Caldo, Ourense), Aquae Celenae (Caldas de Reis, Pontevedra), Aquae Quintiae (Baños de Guntín, Lugo), Aquae Flauiae (Chaves, Portugal), Aquae Bilbilitanorum (Alhama de Aragón, Zaragoza) (Diez de Velasco 1998: 18 y 27) y Aquae Voconiae/Aquae Calidae (Caldes de Malavella, Gerona) (Merino et al. 1994, que aparece en una parte del mapa muy mal conservada, y por tanto podría referirse también a una localidad del sureste de la Galia. Por lo que respecta al norte de África, tenemos las siguientes Aquae que podrian haber estado en la parte perdida de la Tabula: Aquae Dacicae (Sidi Mulay Yakud, cerca de Volubilis, Marruecos), en la Mauritania Tingitana; Aquae Traianae (Hammam Sayala, Túnez), Aquae Persianae (Hammam Lif/Hammam-el-Enf, Túnez), Aquae Aptuccensium (Hammam Biadha, Túnez) y Aquae Calidae Carpitanae (Korbou, Túnez), en África Proconsular; Aquae Sacaritanae (¿?) (Henchir Bischka, Túnez); Aquae Calidae (Hammam Righa, Argelia) y Aquae Sirenses (Bou Hanifia, Argelia) en Mauritania Cesariense; Aquae Flavianae (Henchir el Hammam, Khenchela, Argelia) y Aquae Novae (¿?) en Numidia (Jouffroy 1992: 87-99). En cuanto a Britania: Aquae Sulis (Bath). 
y otras como Constantinopolis, que no puede ser anterior a principios del siglo IV d.C. Aparecen representados, con distintas viñetas, ciudades, puertos, horrea, Aquae, castella, etc, y las tres grandes ciudades a las que ya hemos aludido (Roma, Constantinopla y Antioquía), simbolizadas por figuras entronizadas, así como las cadenas montañosas (en perfil tumbado), los bosques, los ríos y los mares.

La Tabla de Peutinger es básicamente un mapa de caminos, con las calzadas dibujadas en rojo, por medio de líneas rectas. Están representadas aproximadamente unas 70.000 millas romanas. El mapa no sigue ningún tipo de proyección, ni se puede aplicar una escala constante para determinar las distancias de un lugar a otro, aunque éstas suelen estar indicadas en el mapa.

Las proporciones de la Tabla de Peutinger son tales que las distancias de este a oeste están representadas en una escala mucho mayor que las distancias de norte a sur. Por ejemplo Roma parece estar más cerca de Cartago que Nápoles de Pompeya. Mientras que los caminos que siguen la dirección este-oeste están bien representados, los de dirección norte-sur están acomodados a un mapa cuyas dimensiones verticales son muy reducidas. El arquetipo puede muy bien haber sido un rollo de papiro, adaptado de un mapa alargado, como el que podía estar en el Pórtico de Vipsania, en Roma ${ }^{6}$.

\subsection{Las ciudades de Aquae en la Tabula de Peutinger}

Entre las ilustraciones que contiene el mapa, las que representan las ciudades de las Aquae, los baños o balnearios, son las más numerosas y unas de las más Ilamativas. Quizás porque de alguna forma simbolizaban "peace, leisure, and the recovery of health, as well as civilization and his command of technology" (Talbert 2010: 110).

Las Aquae están en primer lugar representadas por lo que Talbert Ilama clase C de viñetas (Talbert 2010: 120),con 36 variantes: el símbolo utilizado es el de un edificio de baños rectangular, presentado oblicuamente desde arriba, para poder mostrar el espacio abierto, o la piscina, pintada de color azul, con techos rojos puntiagudos, y ventanas o puertas, o ambas.

A veces la simple mención en el nombre de la ciudad de la palabra Aqua-Aquae hace que se marque con este símbolo, aunque no sucede así en todos los casos ${ }^{7}$.

Viñetas del tipo $C_{\text {, que representan balnea, aparecen } 35}$ veces en la Tabula, aunque sólo 22 van asociados al nombre Aqua y sus variantes.

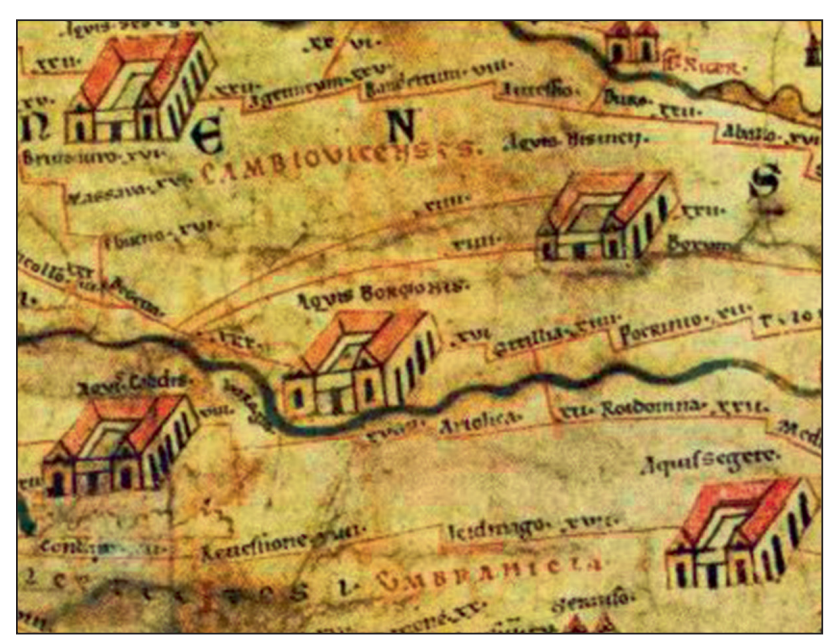

A Figura 2. Parte de la hoja III de la Tabula de Peutinger, que representa Italia, con varias Aquae.

Otras 11 corresponden al tipo con patio cubierto o tejado. De ellas una (Aquis Tatelis) tiene el nombre Aqua y otra es Baiae, famosa ciudad balnear. Otras 4 (de las que 3 corresponden a ciudades de Aquae), tienen otro tipo de viñeta, con patio, pero sin piscina.

Además tenemos otras 9 que contienen el nombre Aqua, pero sin viñeta asociada, siendo por lo tanto 35 los nombres que vamos a examinar.

\subsection{Las estaciones $^{8}$ con Aquae en la Tabula de Peutinger (Talbert 2010c) son:}

- Ad Aqvas (con nombre,con símbolo) 3C5

- Adaqvas (con nombre, sin símbolo) 4B3

- Adaqvas (con nombre, con símbolo,) 5C1

- Adaqvas (con nombre, con símbolo,) 5C3

- Ad aqvas (con nombre, sin símbolo) 6A4

- Adaqvas (con nombre, con símbolo) 6A5

- Adaqvas Albvlas (con nombre, sin símbolo) 4B5

- Ad Aqvas casaris (con nombre, con símbolo) 3C4

- Ad aqvas Hercvlis (con nombre, con símbolo) 3C1

- Aqve Popvlanie (con nombre, con símbolo) 3B2

- Aqvas Apollinaris (con nombre, con símbolo) 4B3

- Aqvas Aravenas (con nombre, sin símbolo) 9B1

- Aqvas labodes (con nombre, con símbolo) 6C1

- Aqvas Passaras (con nombre, con símbolo) 4B1

- Aqvas Regias (con nombre, sin símbolo) 5C1

- Aqvas tavri (con nombre, con símbolo) 4B3

- Aqvas volaternas (con nombre, con símbolo) 3B2

(6) Plinio, N. H. 3, 17: Is namque conplexam eum porticum ex destinatione et commentariis M. Agrippae a sorore eius inchoatam peregit.

(7) "It is presumably deliberate choice, therefore, not accident, that the two occurrences of Adaquas at 5C1 and 5C3 should both be marked by a symbol while Aqvas Regias (5C1) in between them is not. Likewise Aqva viva (4B4) is marked by a symbol, but the same name in $4 A 3$ is not, nor is Adaqvas in 4B3... Likewise it is odd to find Ad Pretorium (405), Ad Horrea (5C2) y Aqva viva (4B4) all marked by category (C) symbol" (Talbert 2010: 120-121).

(8) Se indica si aparece nombre o simbolo en el mapa y el número de segmento y lugar que ocupan en el mismo. 


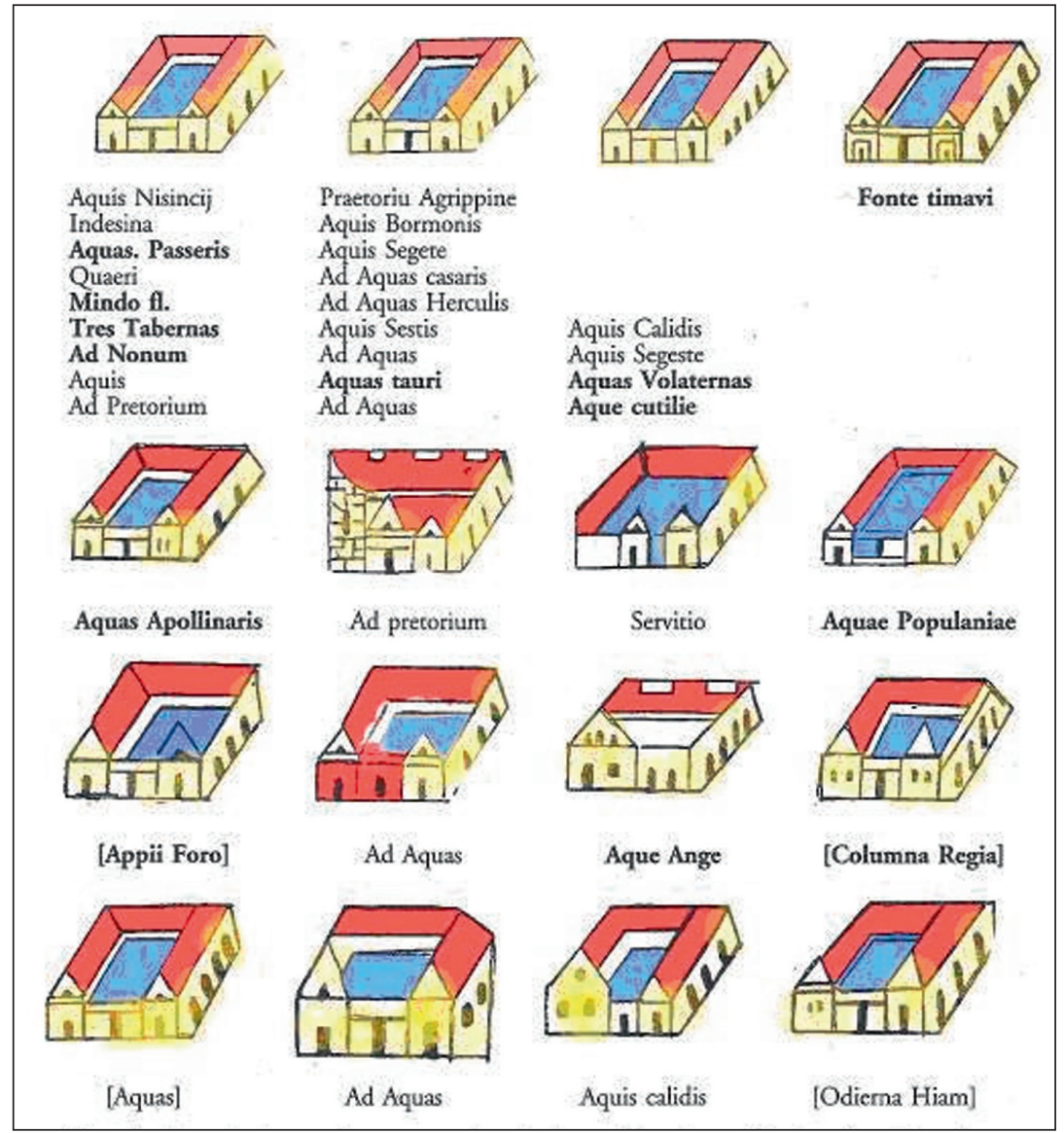

4 Figura 3. Esquema de las mansiones de la Tabula de Peutinger con viñetas del tipo C de Talbert (Allen 2003, fig. 2). Ilustración original tomada de Levi y Levi 1967.
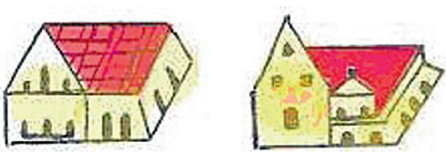

Aquis Tatelis

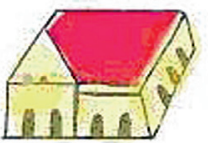

[Baiae]

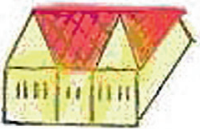

Siclis

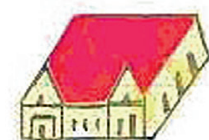

Oplont[i]s

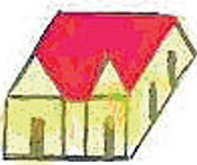

Inaroniac

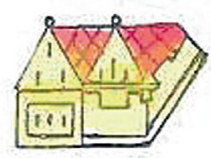

Ad horrea

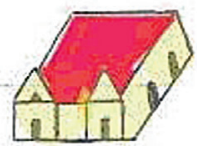

Adteglanum

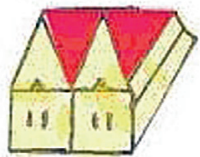

Stanedi
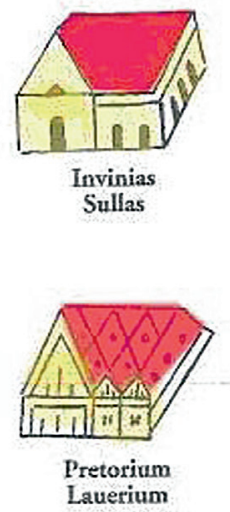

Fig. 3 - Vignetces with roofed or covered courtyard. After Levi and Levi.
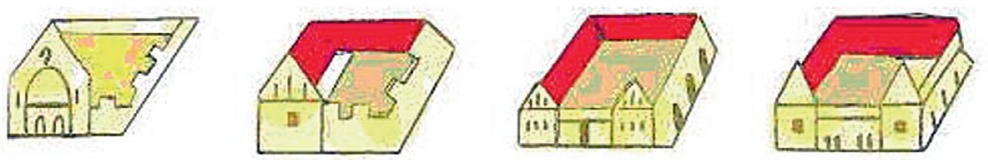

Aquis Thibilimanis
Ad taberna frigida

Ad Aquas
4 Figura 4. Esquema de las mansiones de la Tabula de Peutinger con viñetas del tipo $C$ de Talbert (Allen 2003, fig. 3 y 4). Ilustración original tomada de Levi y Levi 1967. 
- Aqva viva (con nombre, sin símbolo) $4 \mathrm{A3}$

- Aqva viva (con nombre, con símbolo) 4B4

- Aqve Ange (con símbolo, con nombre) 6B1

- Aqve cvtillie (con símbolo, con nombre) 4B4

- Aqvis (símbolo, con nombre) 4C3

- Aqvis [ - ? - ] (con símbolo, con nombre) 1B1

- AQVIS BORMONIS (símbolo, con nombre) 1B4

- Aqvis Calidis (con símbolo, con nombre) 1B4

- Aqvis calidis (con símbolo, con nombre) 7B4

- Aqvis calidis (con símbolo, con nombre) 9B2

- Aqvis Neri (con nombre, no símbolo) 1B3

- Aqvis Nisincii (con símbolo, con nombre) 1B4

- Aqvis Segeste (con símbolo, con nombre) 1A4

- Aqvis Segete (con símbolo, con nombre) 1B5
- Aqvis Tatelis (con símbolo, con nombre) 2B4

- Aqvis Thibilitanis (con símbolo, con nombre) 3C1

- Aqve Frigide (con nombre, sin símbolo) 10B4

- Dissio Aqva Amara (con nombre, sin símbolo) 6C5

\section{CATÁLOGO DE LAS ESTACIONES CON AQVAE EN LA TABULA DE PEUTINGER}

Los datos proceden, sobre todo de Allen (2000) (Túnez); Allen (2003) (Italia); Chevallier (1992) (Galia); Pelletier (1985) (Galia); Díez de Velasco (1998) (Hispania y África); Joffroy (1992) (África), Vega Abeleira (2001) (Britania) y en general de Ihmer (1895) ${ }^{9}$.

\section{Adaquas \\ Ad Aquas. Cerca de Áscoli (ITL) \\ Tipo: con nombre, sin símbolo \\ Segmento del mapa: 4B3 \\ Mansio anterior: X Asclopiceno \\ Mansio posterior: IX Svrpicano \\ Referencias: \\ - BAtlas Ad Aquas 42 E3 \\ - ItMiller 317}

Situada en las cercanías de Ausculum Picenum, en la Vía Salaria.

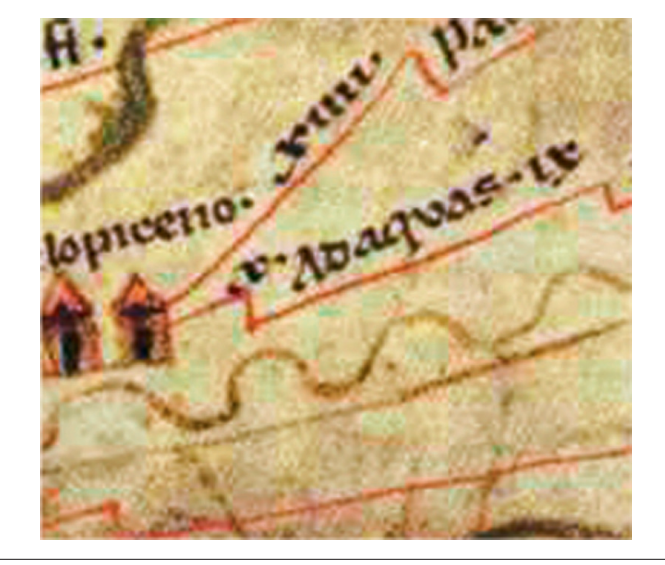

\author{
Ad Aquas \\ Ad Aquas. Hammam Uled Ali bei Souk el Arba (TUN) \\ III $5 \mathrm{u}$ \\ Tipo de símbolo: C28, con nombre \\ Segmento del mapa: $3 \mathrm{C5}$ \\ Mansio anterior: sin cifra de distancia (sin nombre/ \\ ilegible no. 26) \\ Mansio posterior: V Simitv Colonia \\ Referencias: \\ - BAtlas Ad Aquas 32 B3 \\ - ItAnt 43,2 \\ - ItMiller 943 \\ - PaulyWis 4
}

Situada en el África proconsular. Es la mansio Ad Aquas, citada en los itinerarios antiguos, en la vía entre Cartago y Theveste, en un lugar identificado con el topónimo Hammam Uled Ali o Hammam Sidi Ali bel-Kassem (Túnez), donde surgen aguas mesotermales.

(9) BAtlas: Barrington Atlas (Talbert 2000); ItMiller: (Miller 1887); ItAnt: Itinerario de Antonino (Cuntz 1929); PaulyWis: Paulys Realencyclopädie der classischen Altertumswissenschaft (Ihmer 1895); Rav: Anónimo de Rávena (Pinder y Parthey 1860); Ptol: Ptolomeo (Nobbe 1843); CIL: Corpus Inscriptionum Latinarum. 


\section{Adaquas}

Ad Aquas. Korbous ? (TUN) V 1 u

Tipo de símbolo: C22, con nombre

Segmento del mapa: $5 \mathrm{C} 1$

Mansio anterior: XXI Maxvla

Mansio posterior: VIIII Admercvrivm

Referencias:

- BAtlas Ad Aquas 32 F3

- ItMiller 907

- PaulyWis 27

En las cercanias de Cartago, probablemente es el actual hammam de Kourbes Gorb, las antiguas Aquae Carpitanae.

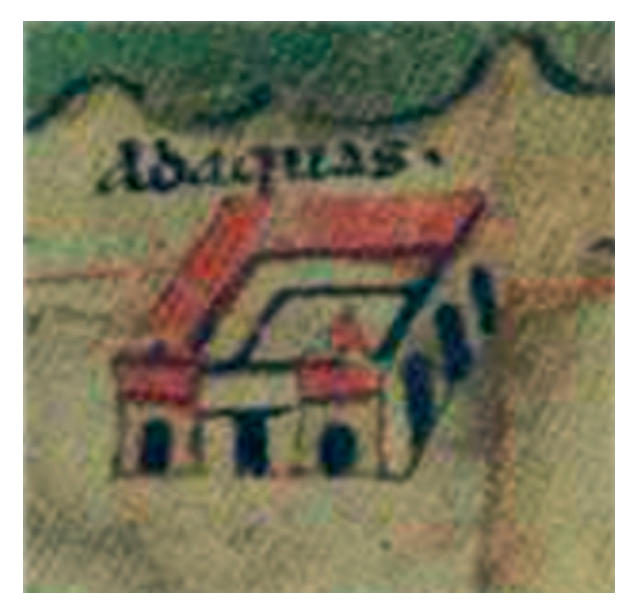

\section{Ad Aquas}

Ad Aquas. Călan (RUM) VI 50

Tipo de símbolo: C29, con nombre

Segmento del mapa: 6 A5

Mansio anterior: XIIII Sarmategte

Mansio posterior: XIII Petris

Referencias:

- BAtlas Aquae 21 E4

- ItMiller 547

- PaulyWis 2

- Rav IV, 7

- Ptol III, 8, 4

Estación en la provincia trajanea de Dacia en la calzada de Sarmizegetusa a Apulum. El lugar que ahora se llama Kis-Kalan y conserva los baños calientes, en donde se han encontrado inscripciones, CIL III, 1403 a 1414.

\section{Ad Aquas}

Aquae Tacapitanae. El Hammă (TUN) V 3 u

Tipo de símbolo: C18, con nombre.

Segmento del mapa: 5C3

Mansio anterior: XVIIII Si[lesv]a

Mansio posterior: XVI Tacape col. "AB AQVIS

TACAPA MILIA XVI".

Referencias:

- BAtlas Aquae Tacapitanae 35 B1

- ItAnt 78,2 Aquas Tacapitanas

- ItMiller 922

- PaulyWis 92

Aquae Tacapitanae, balneario en África, a 18 millas al oeste de Tacape (Gabes) en el camino a Thelepte. 


\section{Ad Aquas Albulas}

Ad Aquas Albulas, La Solfarata, Acque Albule, (ITL) IV $5 \mathrm{~m}$

Tipo: con nombre, sin símbolo

Segmento del mapa: 4B5

Mansio anterior: XVI ROMA

Mansio posterior: Sin cifra de distancia: Tibori

Referencias:

- BAtlas Aquae Albulae 43 C2

- ItMiller 321

- Rav 4, 34, 279

En la Via Tiburtina la mansión Ad Aquas Albulas albergaba un centro termal alimentado por aguas sulfurosas.

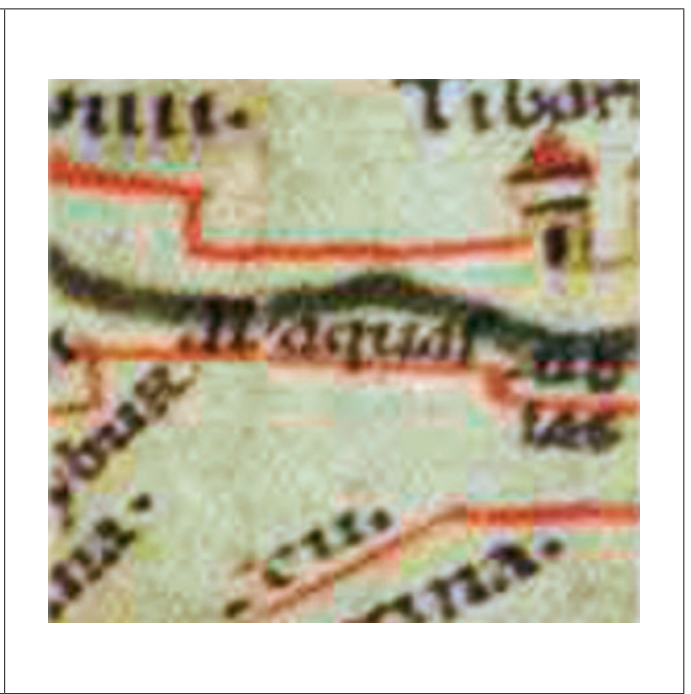

\section{Ad Aquas Caesaris}

Ad Aquas Caesaris, cerca de Tebessa (ARG) III 4 u

Tipo de símbolo: $\mathrm{C2}$, con nombre

Segmento del mapa: $3 \mathrm{C} 4$

Mansio anterior: XVI Ad Mercvrivm

Mansio posterior: VII Theveste

Referencias:

- BAtlas Ad Aquas Caesaris 33 A2

- ItMiller 923

- PaulyWis 23

La estación Ad Aquas Caesaris está a 7 millas al oeste de Theveste. Seguramente estuvo en el lugar actual de Occus o Youk-les-Bains cerca de Tebessa, en el que se han encontrado algunas inscripciones (CIL VIII Sup/ p. 1597).
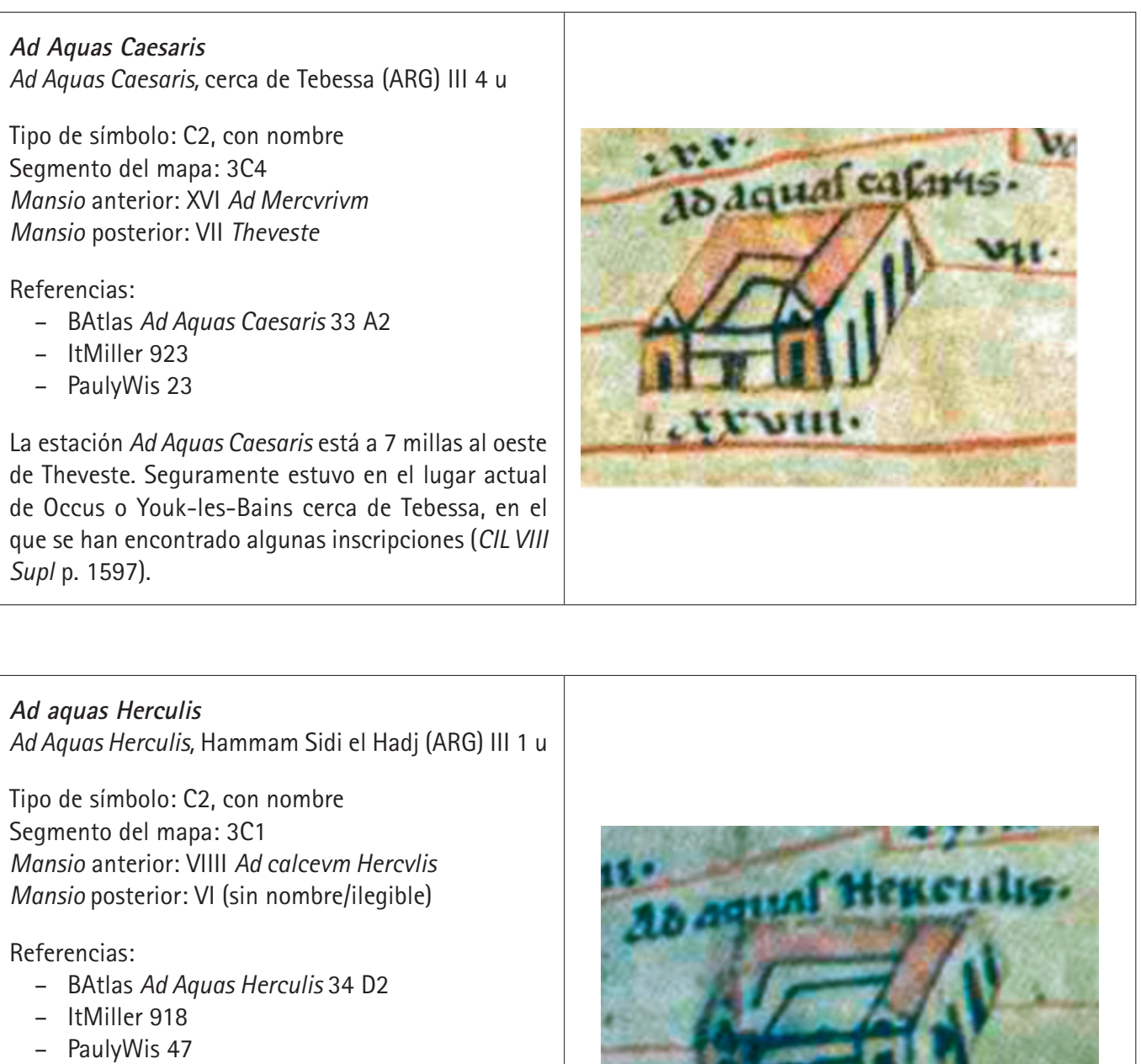

- BAtlas Ad Aquas Herculis 34 D2

- ItMiller 918

- PaulyWis 47

La mansio Ad Aquas Herculis, que está situada en la vía de Lambaesis a Ad Maiores, se ha localizado en Hammam Sidi el Hadj (Argelia). Los restos del balneario romano que explotaba los manantiales sulfurosos mesotermales del lugar son mínimos: una piscina y restos de columnas. Baradez (Baradez 1949: 221) excavó un edificio en las proximidades de la fuente termal actual. 


\title{
Dissio Aqua Amara
}

¿Dissio? ¿Aqua Amara? (LIB?) VI 5 u

Tipo: con nombre, sin símbolo

Segmento del mapa: 6C5

Mansio anterior: XXV Nalad(vs?)

Mansio posterior: XXX Chosol

Referencias:

- BAtlas Dissio Aqua Amara 35, sin localizar

- ItMiller 893-94

Está situada en la gran Syrtis, estación en la vía de Macomades a Tubuctis. Miller piensa que Dissio es una mansio anterior y que falta la distancia, pero es posible también que la Tabula avise de que en Dissio no se podia conseguir más que agua amarga.

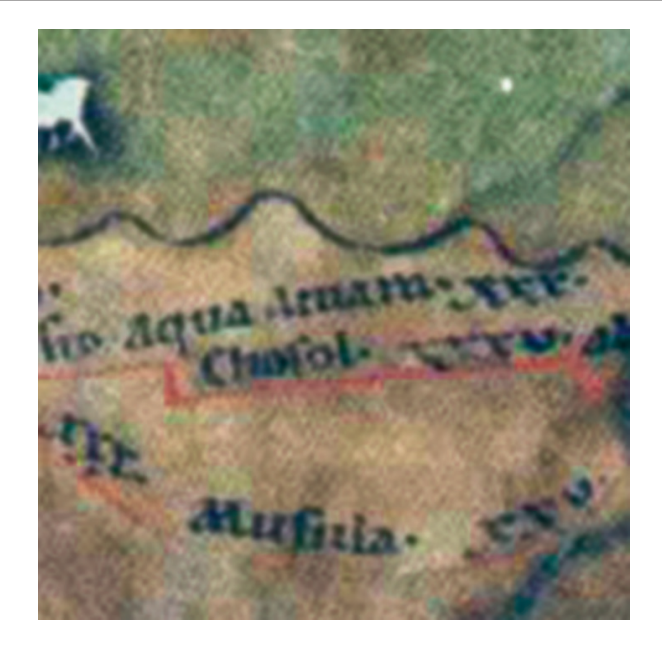

\section{Aqua.Viva}

Aqua Viva, Varazdin (CRO) IV 3 o

Tipo: con nombre, sin símbolo

Segmento del mapa: 4A3

Mansio anterior: X Remista

Mansio posterior: XI Popvlos

Referencias:

- BAtlas Aqua Viva 20 D3

- ItAnt 130,1

- ItMiller 444 Aquaviva

Aqua Viva, mansio situada en Panonia Superior, a 20 millas de Ptuj.

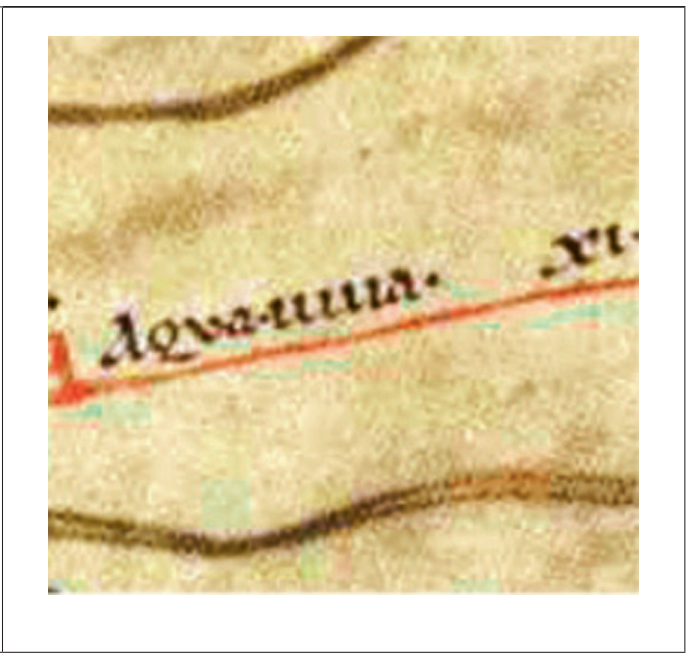

\author{
Aqua Viva \\ Aqua Viva, Acqua viva, cerca de Soracte (ITL) IV $4 \mathrm{~m}$ \\ Tipo de símbolo: C34, con nombre \\ Segmento del mapa: 4B4 \\ Mansio anterior: VII(símbolo, sin nombre, n. ${ }^{\circ} 33$ ) \\ Mansio posterior: Sin cifra de distancia Advicesimv̄ \\ Referencias: \\ - BAtlas Aqua Viva 42 C4 \\ - ItBurd 613,2 \\ - ItMiller 304
}

Aqua Viva, lugar en Etruria en la Via Flaminia entre Rubra y Ocriculum y en la ladera norte del monte Soracte. Hoy lleva el nombre de Acqua Viva. 


\section{Aquis}

Aquae Dacicae, Aïn Kibrit (TUN) IV 3 u

Tipo de símbolo: $\mathrm{C} 1$, con nombre

Segmento del mapa: $4 \mathrm{C3}$

Mansio posterior: VIII Choreva

Mansio anterior: VI Tignica

Referencias:

- BAtlas Aquae? 32 D4

- ItMiller 932

- PaulyWis 39

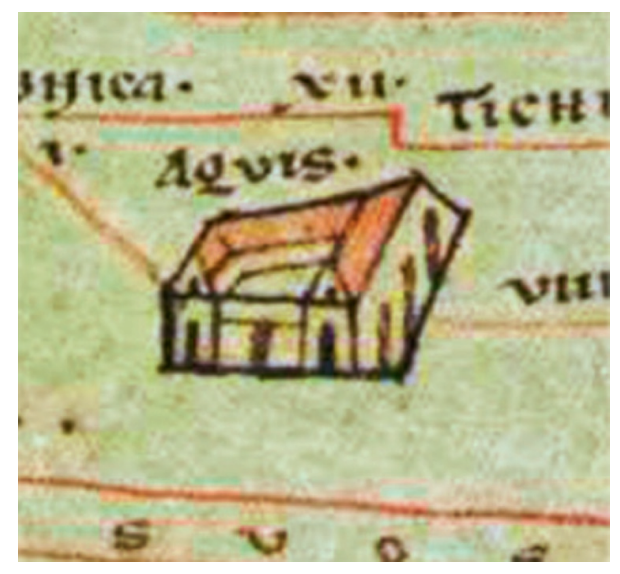

\section{Aque Ange}

Aquae Angae, Bagni di S. Eufemia (ITL) VI $1 \mathrm{~m}$

Tipo de símbolo: C26, con nombre

Segmento del mapa: 6B1

Mansio anterior: XI Temsa (1?)

Mansio posterior: VIII Annicia

Referencias:

- BAtlas 'Aque Ange' 46 sin localizar

- ItMiller 369

\section{Aquas Apollinaris}

Aquae Apollinares, Bagni di Vicarello (ITL) IV 3 m

Tipo de símbolo: C9, con nombre

Segmento del mapa: 4B3

Mansio anterior: XII Tarqvinis

Mansio posterior: VIII Tvrres

Referencias:

- BAtlas Aquae Apollinares 42 C4

- ItAnt 300,3 Aquis Apollinaribus

- ItMiller 298, 247

- PaulyWis 13

Se identifica con los Baños de Vicarello, en la orilla norte del lago de Bracciano, en donde se han encontrado importantísimos hallazgos en el santuario de Apolo (Gasperini, 2008)

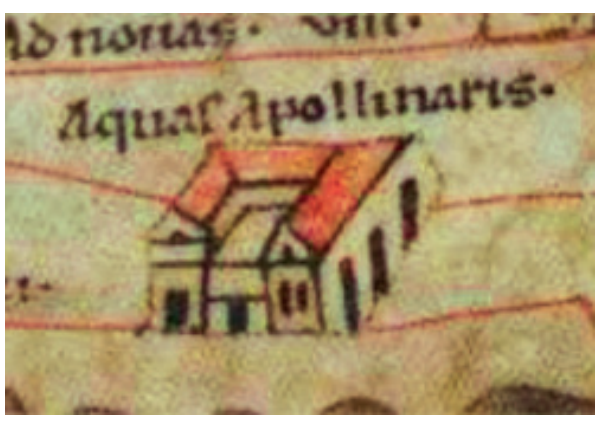



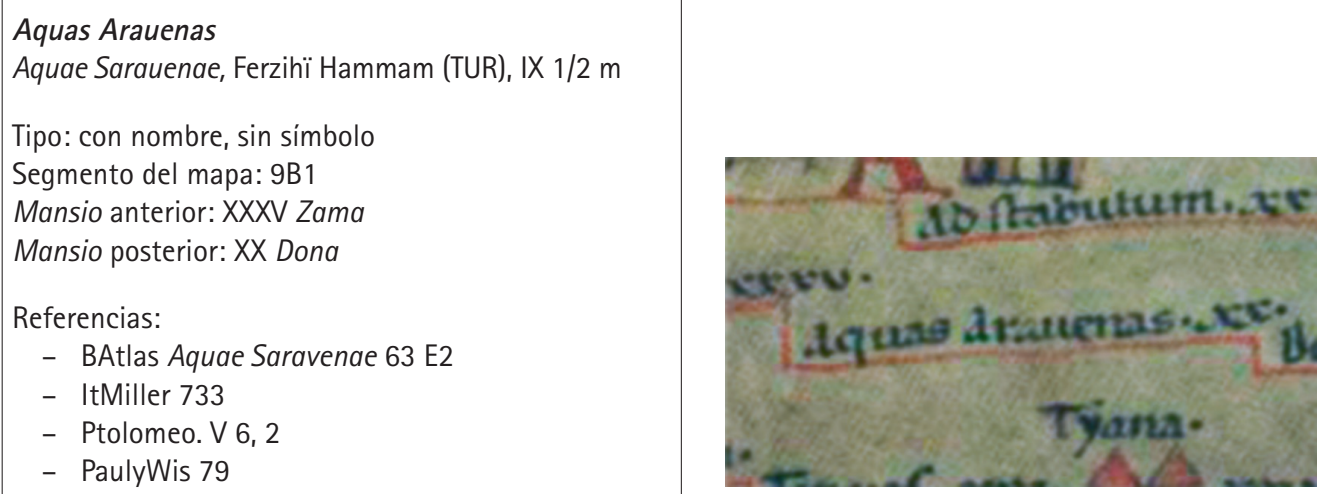

El nombre de la Tabula está equivocado. Aquae Saravenae o Sarvenae,es una ciudad costera a medio camino entre Tavium y Cesarea, hoy Ferzihï Hammam.

\section{Aquis Bormonis}

Aquae Bormonis, Bourbon-l'Archambault (FRA) I 4 m

Tipo de símbolo: C2, con nombre

Segmento del mapa: 1B4

Mansio anterior: XXX Degetia

Mansio posterior: XVI Sitillia

Referencias:

- BAtlas Aquae Bormonis? 18 A3

- ItMiller 101

- PaulyWis 20

Aquae Bormonis, en Aquitania,en la calzada de Autun a Orléans. El lugar tiene el nombre de la deidad local Borvo o Bormo, cuyo nombre deriva de la palabra céltica para las aguas termales.
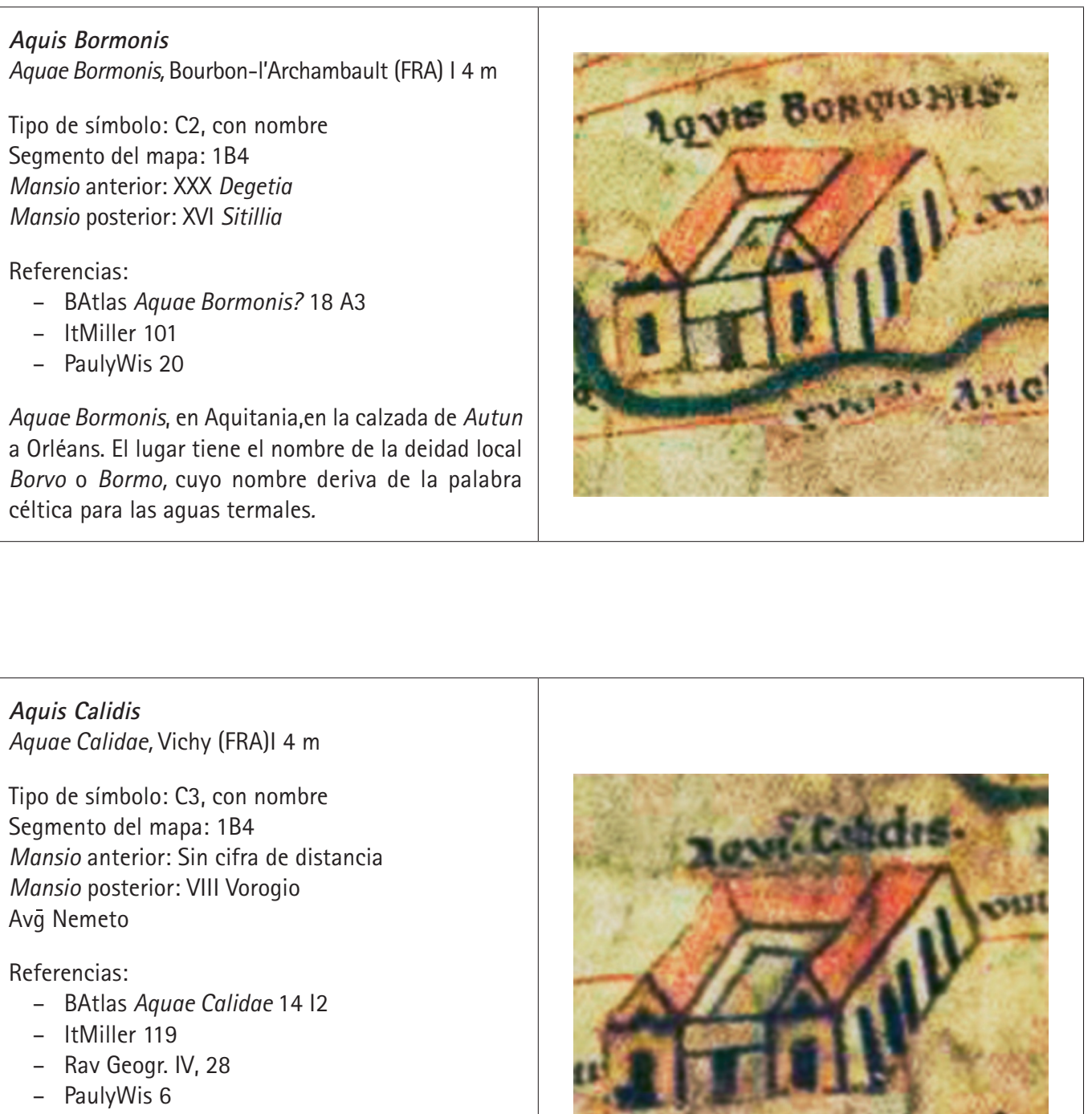

Aquae Calidae, localizada en Aquitania, está situada en la calzada de Augustonemetum a Forum Segusiavorum. 


\section{Aquis Calidis}

Aquae Calidae, cerca de Rumkoci, (BUL)VII 3 m

Tipo de símbolo: C30, con nombre

Segmento del mapa: 7B4

Mansio anterior: L Cabilis

Mansio posterior: XII Ancialis

Referencias:

- BAtlas Aquae Calidae 22 E6

- ItMiller 590

- PaulyWis 24

Situada en Tracia, a 12 millas al oestede Anchialos, en el Ponto Euxino, en el camino a Philippopolis. Hubo una fuente mesotermal, para bañarse y beber, con un próspero balneario junto al mar en el período bizantino.

\section{Aquis Calidis}

Aquae Calidae, Çiftehan (TUR), IX 2m

Tipo de símbolo: $\mathrm{C} 1$, con nombre

Segmento del mapa: 9B2

Mansio anterior: XXXIX Tyana

Mansio posterior: XII Padvando

Referencias:

- BAtlas Aquae Calidae 66 F1

- CIL VI 5076 Ad Aq(uas) Calida(s) 664

- PaulyWis 25

Aquae Calidae estaba situada en Capadocia, en la calzada de Tiana a Cesarea.

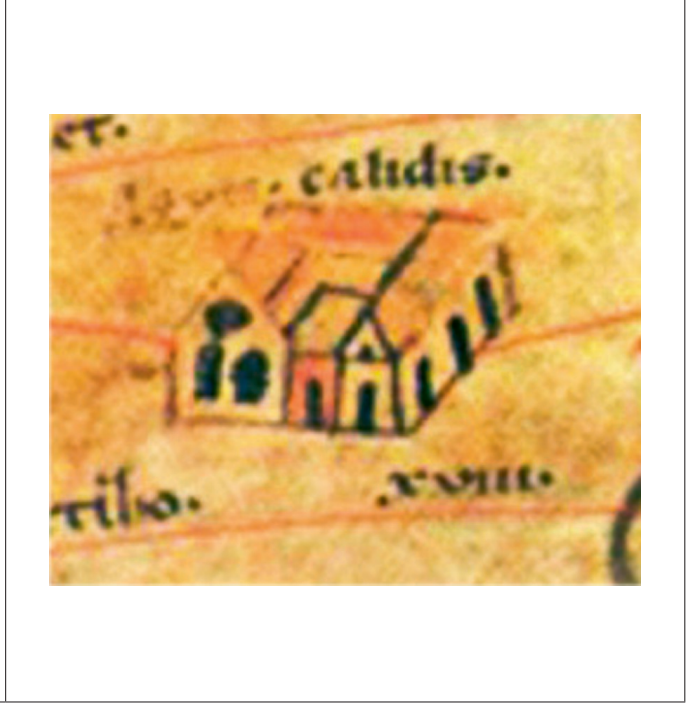

\section{Aque Frigide}

Aquae Frigidae, Meiacarire (TUR), X 4 m

Tipo: con nombre, sin símbolo

Segmento del mapa: 10B4

Mansio anterior: XVII Sammachi

Mansio posterior: Sin cifra de distancia, Arcamo.

Referencias:

- BAtlas Meiacarire/*Aquae Frigidae 89 C3

- ItMiller 741

- PaulyWis 43

Aquae Frigidae en Mesopotamia, al noroeste de Singara de Nisibis. Citada por Amiano Marcelino, 18, 6,1 y 16.

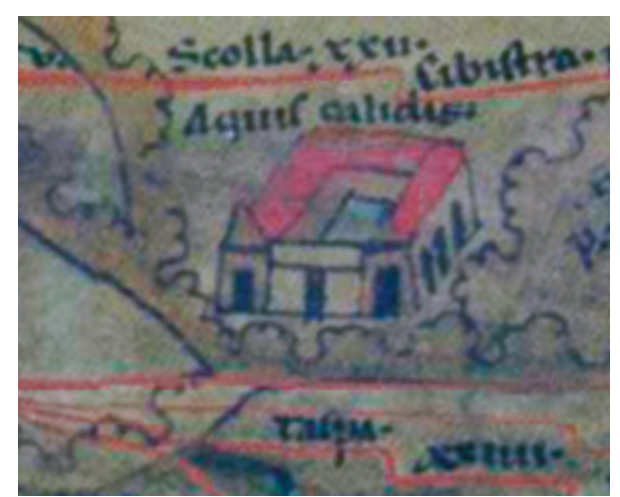



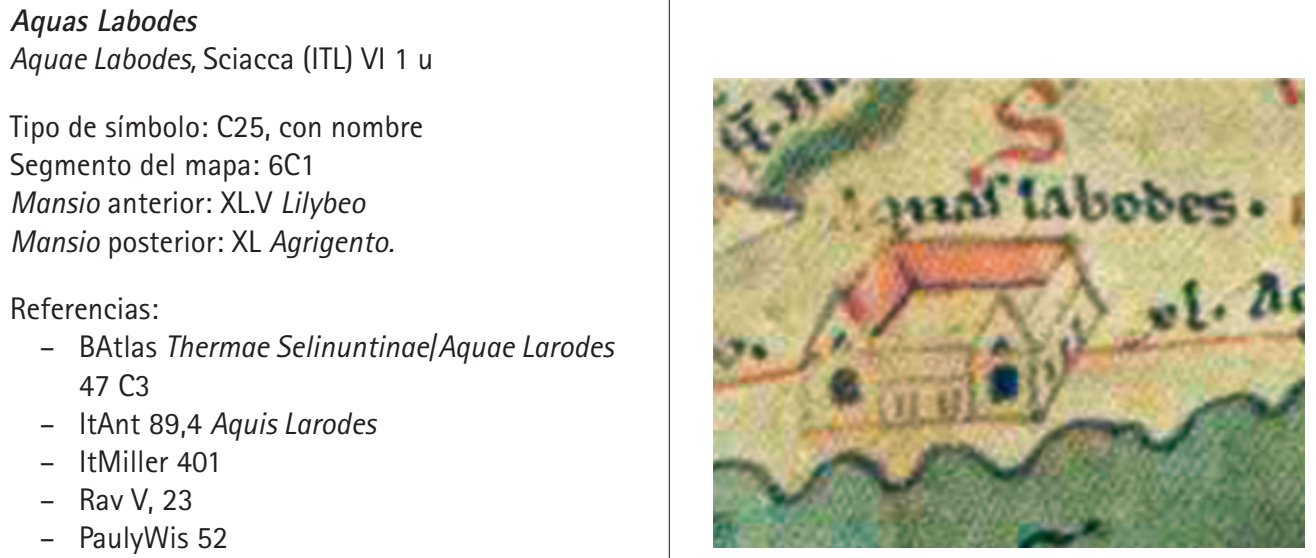

Baños minerales calientes y salados, en Sicilia.

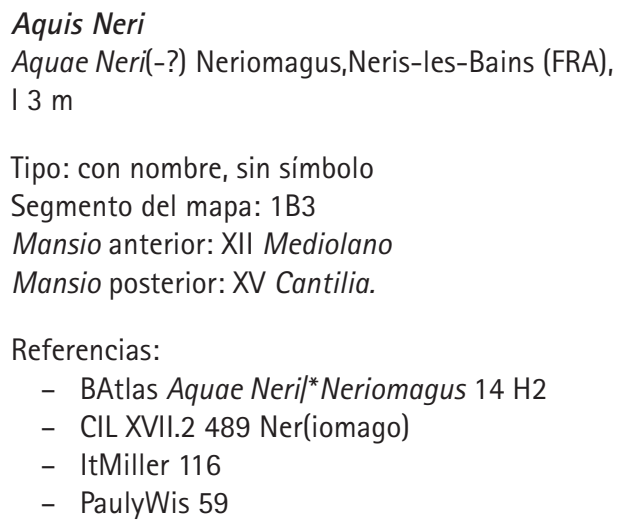

Situados en Aquitania, en la vía de Augustonemetum a Lemunum. Inscripción dedicada al Deus Nerius.

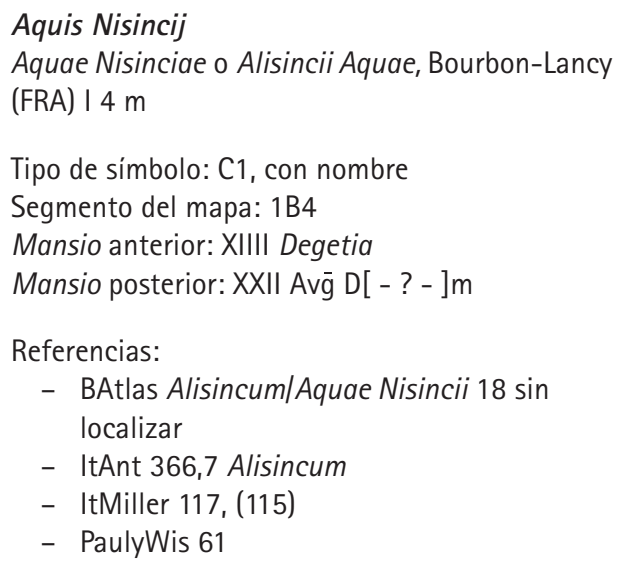

En la Lugdunense. Entre Autun y Decise. Apolo era el dios protector de las aguas termales. 


\section{Aquas Passaris}

Aquae Passeris, Bagni Giasinelli (ITL) IV 1 m

Tipo de símbolo: $\mathrm{C} 1$, con nombre

Segmento del mapa: 4B1

Mansio anterior: VIIII Volsinis

Mansio posterior: XI Foro Cassi

Referencias:

- BAtlas Aquae Passeris 42 C4

- ItMiller 290

- PaulyWis 66

Aquae Passeris, en Etruria, a 11 millas de Forum Cassii y 9 de Volsinii (Bolsena).

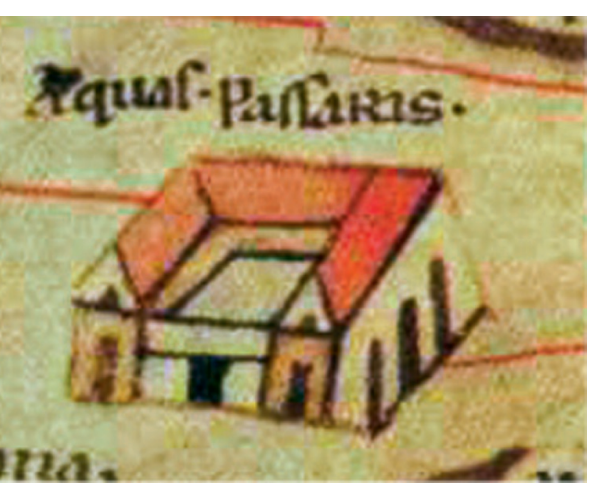

\section{Aque Populanie}

Aquae Populaniae, ¿Bagni della Leccia? (ITL)III $2 \mathrm{~m}$

Tipo de símbolo: C17, con nombre

Segmento del mapa: 3B2

Mansio anterior: VII Maniliana

Mansio posterior: XXXIII Adsextvm

Referencias:

- BAtlas Aquae Populoniae 41, sin localizar

- ItMiller 294-95

- Rav IV 36

- PaulyWis 73

Estaban en Etruria, entre Saena y Populonium, tal vez el Bagni della Leccia, a $12 \mathrm{~km}$ al norte de Massa Marítima. En la excavación cerca de Sasso Pisano,del lugar llamado en el medievo Bagno del Re y Bagno S.Quilico, en donde brotan aguas sulfurosas y aluminosas calientes, se ha encontrado un gran complejo termal etrusco y romano. En los ladrillos aparece la marca de la ciudad de Populonia.

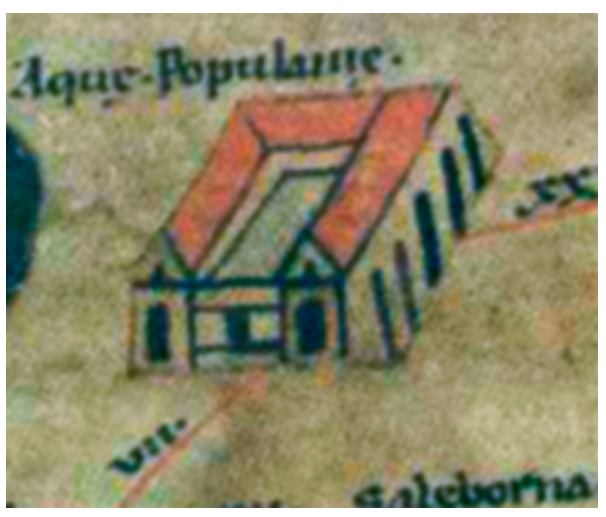

\section{Aquas Regias}

Aquae Regiae, Henchir Khatera o Henchir Babuscha (TUN) V 1 u

Tipo: con nombre, sin símbolo

Segmento del mapa: 5 C1

Mansio anterior: XIIII AGGAR

Mansio posterior: XVI Terento

Referencias:

- BAtlas Aquae Regiae 33 E1

- ItAnt 53,2 Aquis Regis

- Rav. III,5

- ItMiller 928

- PaulyWis 76

En África proconsular. Es una población de cierta importancia, citada como Aquisegiensis civitas en Victor Vitensis III, 5. 


\section{Quaeri}

¿Aquae Risani?, Koper (YUG) III 5/IV 10

Tipo: con nombre, con símbolo, C1.

Segmento del mapa: 4A1

Mansio anterior: (sin distancia): Parentio

Mansio posterior: (sin distancia) Silvo.

Referencias:

- ItMiller 312

En las excavaciones del 2000-2001, en la orilla derecha del Risano, frente a Sermino, apareció una gran villa, que podría ser la localidad de la Tabula (Župančič, 2005).

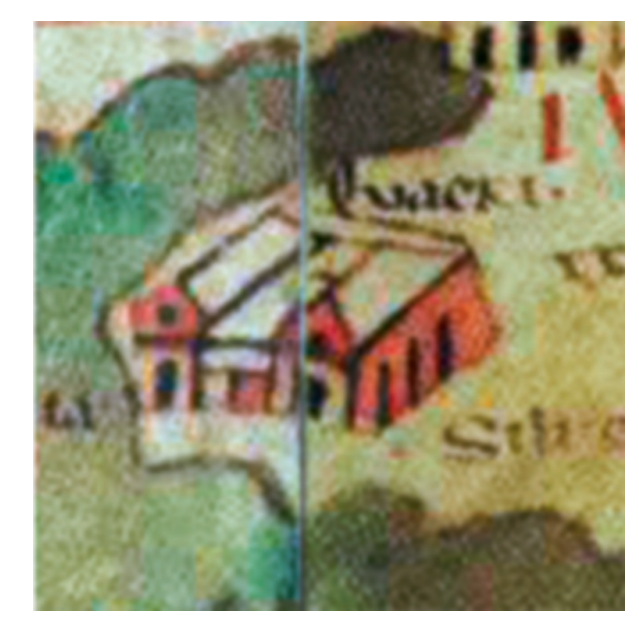

\section{Aquae Segeste}

Aquis Segeste, Ferrières (FRA) $14 \mathrm{~m}$

Tipo de símbolo: C3, con nombre

Segmento del mapa: $1 \mathrm{~A} 4$

Mansio anterior: XXII Fines

Mansio posterior: XXII Agetincrm

Referencias:

- ItMiller 117

- RE Aqua 80

Aquae Segesta estaba en la calzada entre Agetincum y Cenabum.

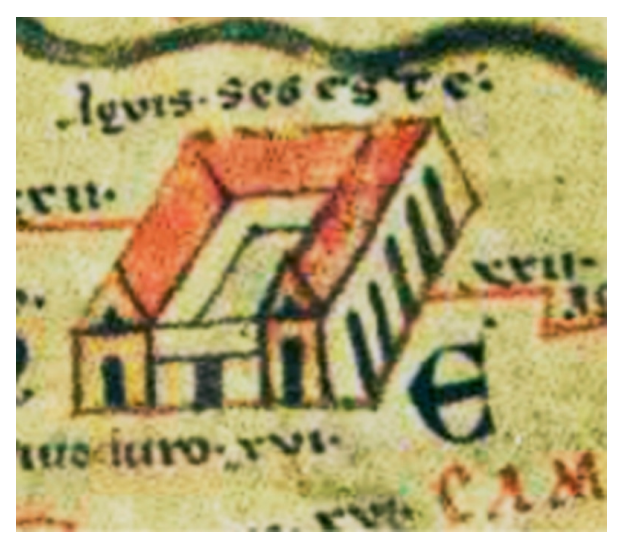

\section{Aquis Segete}

Aquae Segetae, Bains de Saint Galmier, Greppo (FRA) I $5 \mathrm{~m}$

Tipo de símbolo: $\mathrm{C} 2$, con nombre

Segmento del mapa: 1B5

Mansio anterior: XVII Icidmago

Mansio posterior: VIIII Foro Segvstavarv̄

Referencias:

- BAtlas Aquae Segetae 17 C2

- ItMiller 94

- Rav. IV 26

- PaulyWis 82

La diosa romana de la siembra Segetia (Segesta) no parece que tenga nada que ver con el nombre de la localidad.

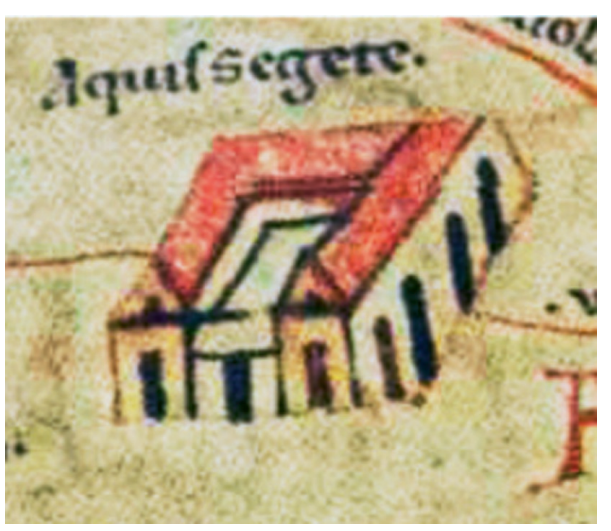


[ - ? - ]estis

Aquae Sextiae, Aix-en-Provence (FRA) II $1 \mathrm{~m}$

Tipo de símbolo: C2, con nombre

Segmento del mapa: 2B1

Mansio anterior: XVIII Pisavis

Mansio posterior: XL. IIII Reis Apollinaris

Referencias:

- BAtlas Col. Aquae Sextiae 15 E2

- ItAnt 298,5 Aquis Sextis

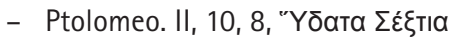

- ItMiller 133,130 Aquis Sestis

- PaulyWis 85

Su antiguo nombre era Aquae Sextiae / Col. Augusta Aquae Sextiae / Civitas Aquensium

En la vía de Forum Iulii a Arelate. Tuvo aguas

termales famosas: "Propter aquarum copiam e caldis frigidisque fontibus" (Epit. Tito Livio. LXI)

\author{
Aquis Tatelis \\ Aquae Statiellae, Acqui Staziella (ITL) II 4 m \\ Tipo de símbolo: $\mathrm{C4}$, con nombre \\ Segmento del mapa: 2B4 \\ Mansio anterior: XXII Crixia \\ Mansio posterior: X Alba Pompeia \\ Referencias: \\ - BAtlas Aquae Statiellae 39 C4 \\ - ItAnt 294,7 Aquis \\ - Strab V 217 \\ - CIL V 7153, Aquenses Statie/(li) \\ - ItMiller 253 \\ - PaulyWis 89
}

Ciudad de la Liguria con baños calientes. La menciona Plinio el Viejo, N. H. XXXI

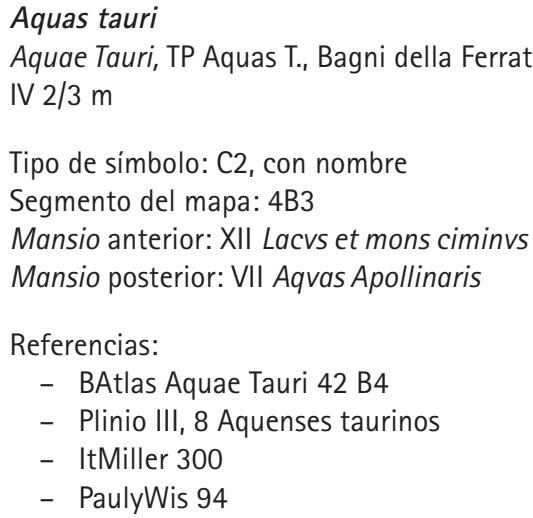

- BAtlas Aquae Tauri 42 B4

- Plinio III, 8 Aquenses taurinos

- ItMiller 300

- PaulyWis 94

A $3 \mathrm{~km}$ de Centumcellae, en Etruria. Tenía manantiales de agua mineral caliente.
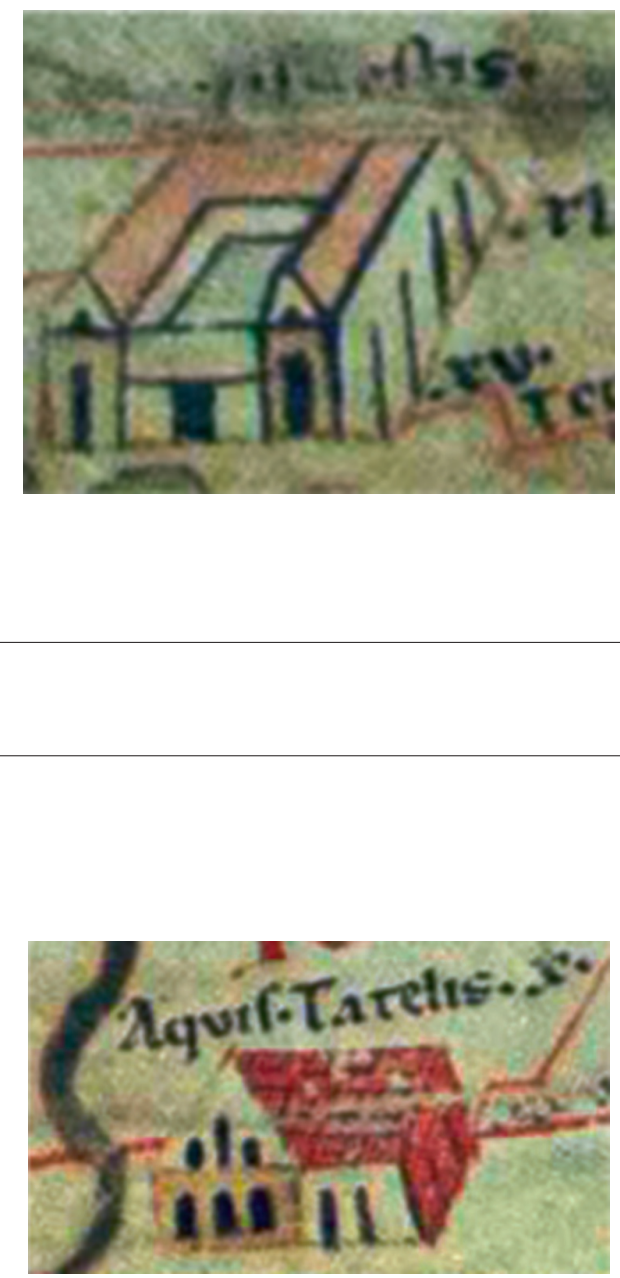


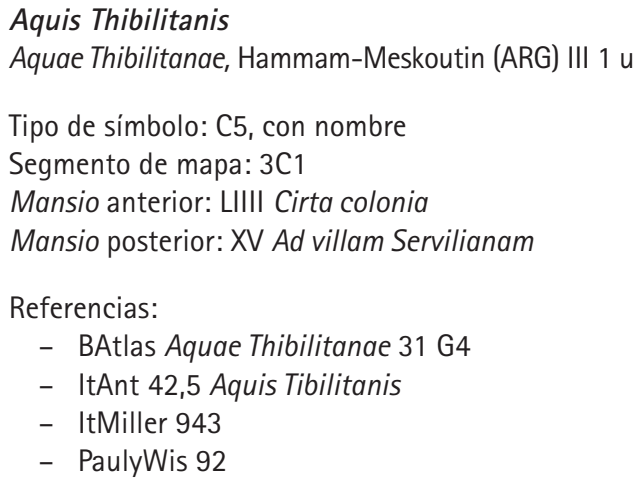

En el camino de Cirta a Hippo Regius. Al norte de las ruinas de la antigua Thibilis.

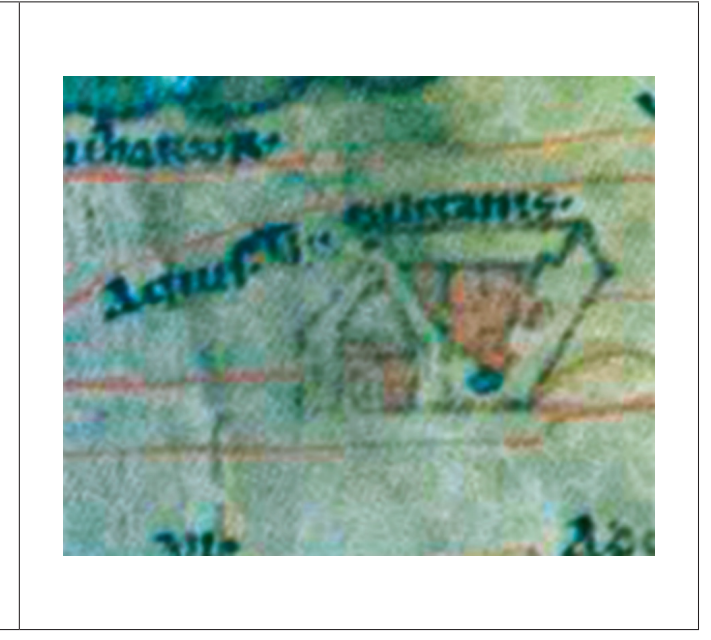

Aquis. Voconi

Aquae Voconae, Caldas de Malavella, Gerona

(ESP) I $1 \mathrm{~m}$

Tipo de símbolo: C33, con nombre

Segmento del mapa: 1B1

Mansio anterior: No hay

Mansio posterior: Sin cifra de distancia (símbolo, no nombre, n. ${ }^{\circ}$ )

Referencias:

- BAtlas Aquae Convenarum 25 F2

- ItAnt 457,7 Aquis Convenarum

- ItMiller 98 Aquis Convenarum

- PaulyWis 99

Miller creía que podia tratarse de Aquis Convenarum, pero parece más lógico que ambas palabras pertenezcan a la misma mansio.

\section{Aquas Volaternas}

Aquae Volaterranae, Bagno a Morbo, Montecerboli (ITL) III $2 \mathrm{~m}$

Tipo de símbolo: $\mathrm{C} 3$, con nombre

Segmento del mapa: 3B2

Mansio anterior: Sin cifra de distancia Vadis

Volateris.

Mansio posterior: No hay.

Referencias:

- ItMiller 294

- RE Aqua 100

Baños termales de Morba, a $25 \mathrm{Km}$ al sur de Volterra.
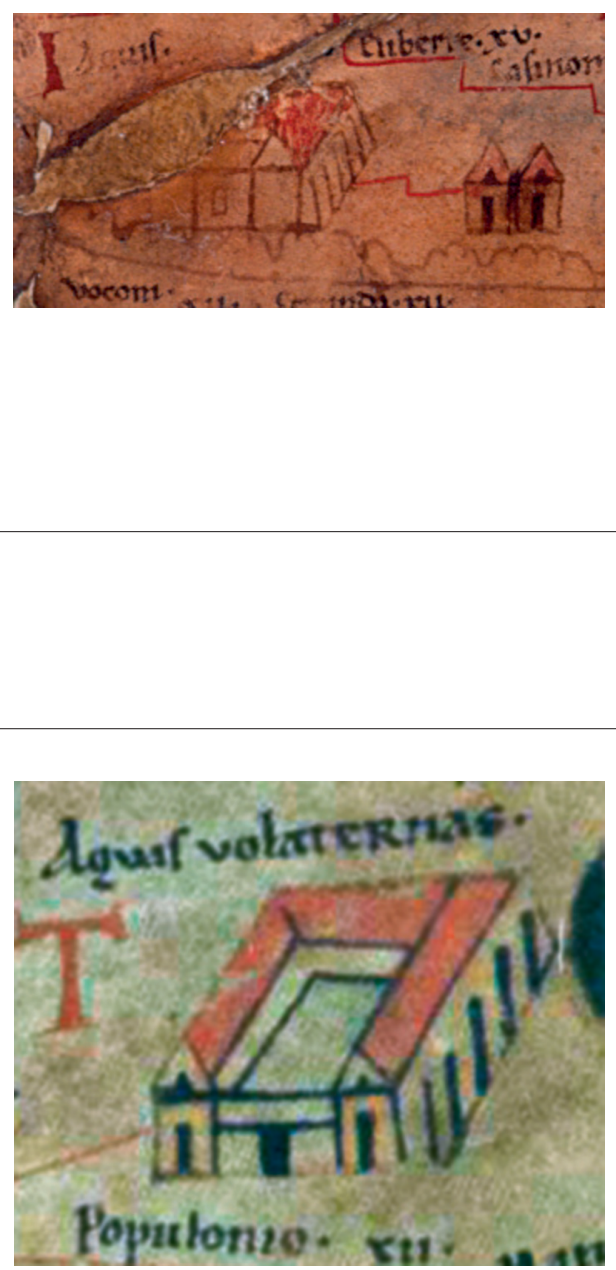


\section{CONCLUSIONES}

Esta somera descripción introductoria de las estaciones que, en la Tabula de Peutinger, llevan el nombre de Aquae, pone de manifiesto la importancia que le dio el autor del mapa a estos lugares: balnearios y ciudades balneares que habian crecido en torno a ellos, probablemente destacados en función del uso terapeútico de sus aguas. Es Ilamativo su gran número y el tamaño más grande de los símbolos asociados a ellos. Aunque R. Chevallier (1997, nota 5) considera que no hay por qué hacer de la Tabula de Peutinger una guía termal, no hay duda de que se trataba de señalizarlas en el mapa, destacándolas con un símbolo para su mejor localización. En todo caso, resulta razonable considerar que la utilización del plural Aquae parece estar reservado a los lugares con aguas termales o con propiedades curativas. Presentan la característica común de haberse desarrollado en torno a una fuente de aguas medicinales, normalmente fuera de las aglomeraciones urbanas y con una finalidad diferente a la de los edificios termales construidos en ciudades o villae, tanto públicos como privados.

\section{BIBLIOGRAFÍA $^{10}$}

ABAD, M. 1992: "La moneda como ofrenda en los manantiales", Espacio, Tiempo y Forma II, 5: 133-191.

Abascal, J. M., Almagro GorbeA, M. y LoRrio, A. J. 1977: "Las termas monumentales de Segobriga", Revista de Arqueología 195: 38-45.

AlLEN, T. J. 2000: "Taking the Waters: A new survey of spas in Tunisia", Classical Association of the Pacific Northwest. Bulletin, 31, 1 http://darkwing.uoregon.edu/ mwilson/capn/CAPNbulletins/ Vol31.1.html

- 2003: "Roman healing spas in Italy: the Peutinger map revisited", Athenaeum, 91, 2: 403-415.

Almagro GorbeA, M. y ABASCAL, J. M. 1999: Segobriga y su conjunto arqueológico, Madrid.

Álvarez, J. M. 1972: "Las termas romanas de Alange", Habis 3: 267-290.

Baradez, J. 1949: Fossatum Africae. Recherches Aériennes sur l'organisation des confins Sahariens a l'Epoque Romaine, Arts et Métiers Graphiques, Paris.

BarATte, F. 1992: "La coupe en argent de Castro Urdiales", Caesarodunum. XXVI: 43-54.

Beltrán, A. 1954: "Los hallazgos del balneario de Panticosa (Huesca)", Caesaraugusta 5: 196-200.

Biblotheca Augustana, Tabula Peutingeriana, Facsimil de Müller, http://www.hs-augsburg.de/ harsch/Chronologia/Lspost03/Tabula/ tab_pe00.html

Bosı, L. 1983: La Tabula Peutingeriana: una descrizione pittorica del mondo antico, Rimini.

BouRDY, F. 1992: "Du bon usage des bains d'après Oribase" Caesarodunum, XXVI: 31-38.

Brodersen, K. 2003: "Die Tabula Peutingeriana: Gehalt und Gestalt einer alten Karteund ihrer antiken Vorlage", en: D. Unverhau (ed.), Geschichtsdeutung auf alten Karten: Archäologie und Geschichte. (Wolfenbütteler Forschungen 101) Wiesbaden: 289-297.

CheVallieR, R. (ed.) 1992: "Les eaux thermales et les cultes des eaux en Gaule et dans les provinces voisines", Actes du Colloque (28-30 Septembre 1990), Caesarodunum XXVI.

- 1997: Les voies romaines, Paris.

CIURLeTI, G. 1991: Tabula Peutingeriana, Codex Videbonensis. Completa di tutti gli 11 segmenti nel formato originale $76 \times 42$, Trento.

Costa, A., Palahl, LL. y Vivó, D. (eds.) 2011: AOVAE SACRAE. Agua y sacralidad en la Antigüedad, Girona.

CunTz, O. 1929: Itineraria Romana. Volumen prius. Itineraria Antonini Augusti et Burdigalense, Stuttgart.

Diez de Velasco, F. 1992: "Divinités des eaux termales dans le NordOuest de la Provincia Tarraconensis et dans le Nord de la Provincia Lusitania", Caesarodunum XXVI: 133-149.
- 1998: "Termalismo y religión. La sacralización del agua termal en la Península lbérica y el norte de África en el mundo antiguo", ILU. Revista de Ciencias de las religiones, monografias 1, Madrid.

Diez de Velasco, F., Miró, C. y Peréx, M. J. 1992: "Introducción bibliográfica al termalismo antiguo en la Península Ibérica", Espacio, Tiempo y Forma. Serie 2. Historia Antigua 5: 593-604.

Dupré, N. y Peréx, M. J. 1992: "Thermalisme et religion dans le Nord de I'Hispania", Caesarodunum XXVI: 151-169.

FABRE, G. 2004: "Divinidades y cultos relacionados con las aguas", Aqua Romana. Técnica humana y fuerza divina, Catálogo de la Exposición del Museu de les Aigües, Bacelona: 146-160.

FRADE, H. 1993: "As termas medicinais de época romana em Portugal", Actas do ll Congresso Peninsular de Historia Antiga (Coimbra 18-20 October 1990): 873-916.

GASPERINI, L. 2008: "El tesoro de Vicarello. Un gran descubrimiento arqueológico del siglo XIX", Gerion 26, 2: 91-102.

GozALBES, E. 1997: "Los baños y la curación de Augusto en Tarraco", PeréX, M. J. (ed.) Termalismo Antiguo: 241-245.

IHMER, K. 1895: "Aquae", Stuttgart: 294-307.

Jansoon 1741: Tabula Itineraria ex illustri Peutingerorum Bibliotheca quae Mugistae Vindelicorum beneficio Marci Velseri Septem Veri Augustani in lucem edita.

Joffror, H. 1992: "Les Aquae africaines" en R. CHEVALLIER, Les eaux thermales et les cultes des eaux en Gaule et dans les provinces voisines, Caesarodunum XXVI: 87-99.

LEVI, A. y LEVI, M. 1967: Itineraria picta: Contributo allo studio della Tabula Peutingeriana, Roma.

Martinez Saura, F. y Montero, S. 1997: "La balneoterapia en la obra de Celso", PerÉx, M. J. (ed.) Termalismo Antiguo: 235-240.

Merino, J., Nolla, J. M. y SAntos, M. 1994: "AQUAE CALIDAE. Presència romana a la Selva", Estudis i textos, 3, Santa Coloma de Farners (la Selva).

MILLER, K. 1916: ItIneraria Romana. Römische Reisewege an der Hand der Tabula Peutingeriana, Stuttgart.

- 1887: Die Weltkarte des Castorius: genannt die Peutingersche Tafel, Ravensburg, Maier.

MıRó, C. 1992: "La arquitectura medicinal termal en época romana en Catalunya. Las termas de Caldes de Montbui como ejemplo", Espacio, Tiempo y Forma II, 5: 255-275.

Miró, M. T. y Mıró, C. 1997: "Los tratamientos hidroterápicos en los textos clásicos", PERÉX, M. J. (ed.) Termalismo Antiguo: 211-216.

Moltó, L. 1992: "Tipos de aguas minero-medicinales en yacimientos arqueológicos de la Península Ibérica", Espacio, Tiempo y Forma II, 5: 211-228.

MoRA, G. 1981: "Las termas romanas en Hispania", Archivo Español de Arqueología, 58: 37-89.

${ }^{(10)}$ Incluimos la bibliografía citada y aquella actualizada de mayor interés sobre el tema del artículo. 
- 1992: "La literatura médica clásica y la arquitectura de las termas medicinales", Espacio, Tiempo y Forma II, 5: 121-131.

Motilla, G., EgeA, A. y Gallardo, J. 2004: "La sacralidad de las aguas. El caso de los santuarios romanos de Fortuna (Murcia)", Aqua romana. Técnica humana y fuerza divina, Catálogo de la Exposición en el Museu de les Aigües, Barcelona: 162-176.

NobBe, C. F. A. 1843: Claudii Ptolemaei Geographia. 3 vols. Leipzig: Carolus Tauchnitus.

Oró, E. 1997. "Las aguas mineromedicinales en la medicina de la Antigüedad", PerÉx, M. J. (ed.) Termalismo Antiguo: 229-234.

PANAITE, A. y CîRJAN, R. 2004: "Juridical Status of the Roman Cities and their Representation in Late Roman Cartography", Quaderni della Casa Romena, 3: 21-30.

Pelletier, A. (ed.) 1985: La médecine en Gaule. Villes d'eaux, sanctuaires des eaux, Paris.

Peréx, M. J. et alii 2006: "The Use of Water for Health Purposes in Roman Hispania", en Cura Aquarum in Jordania. 13th International Congress on Water Management and Hidraulic Engineering in the Mediterranean Region, Leuven-Paris-Dudley: 441-445.

- (Coord.) 1992: Termalismo Antiguo. Aguas mineromedicinales, termas curativas y culto a las aguas en la Península lbérica, Actas de la mesa redonda (Madrid 28-30 Noviembre 1991), Espacio, Tiempo y Forma II, 5.

- (ed.) 1997: Termalismo Antiguo, Actas dell Congreso Peninsular (Arnedillo, La Rioja, 3-5 Octubre 1996), Casa de Velázquez / Universidad Nacional de Educación a Distancia, Madrid.

PeutingeR's TABLE http://www.cambridge.org/us/talbert/talbertdatabase/ all-alphabetical.html
Pinder, M. y Parthey, G. 1860: Ravennatis Anonymi Cosmographia et Guidonis Geographica, Berlin.

PLINIO EL VIeJo 2007: Historia Natural, ed. de Cantó et altres, Madrid, Cátedra.

Rodriguez Morales, J. 2011: "Los nombres de las mansiones con $a d+a c u s a t i v o$ en las fuentes itinerarias", El Nuevo Miliario 12: 4254.

StUART, P. 1999: De Tabula Peutingeriana, Nijmegen.

TABUla PeutingerIana, http://www.tabula-peutingeriana.de/tp/tpx.html

TALBERT, R. T. (ed.) 2000: Barrington Atlas of the Greek and Roman World (with Map-by-Map Directory on CD-ROM)

- 2007a: "Peutinger's Roman Map: the Physical Landscape Framework", Wahrnehmung und Erfassung geographischer Raumein der Antike, Michael Rathmann (ed.), Mainz am Rhein: 221-230.

- 2010b: Rome's World. The Peutinger Map Reconsidered, Cambridge University Press.

- 2010c: Peutinger map: seamless whole, in color, with overlaid layers (Map A). http://peutinger.atlantides.org/map-a/

- 2010d: Peutinger Map with names and Features (links with Map A), with Commentary and User's Guide (= Appendix 7)

http://www.cambridge.org/us/talbert/talbertdatabase/prm.html

Vega Abeleira, T. 2001: "Baños y asentamientos militares romanos en Britannia", Minius. Revista do Departamento de Historia, Arte eXeografía 9: 51-76.

WEBER, E. 1976: Tabula Peutingeriana. Codex Vindebonensis 324: Graz.

Župančıč, M. 2005, "Il Territorium Caprense, la Via Flavia e il pluteo con decorazioni ad intreccio attorno all'800'", Acta Histriae, 13, 1: 225-236. 